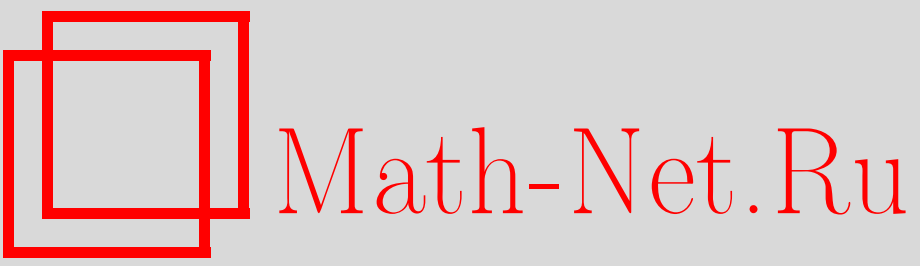

А. М. Боярский, Т. В. Скрыпник, Сингулярные орбиты коприсоединенного представления евклидовых групп, УМН, 2000, том 55, выпуск 3, 169-170

DOI: https://doi.org/10.4213/rm296

Использование Общероссийского математического портала Math-Net.Ru подразумевает, что вы прочитали и согласны с пользовательским соглашением

http://www.mathnet.ru/rus/agreement

Параметры загрузки:

IP: 52.6 .47 .48

26 апреля 2023 г., 14:54:31 


\title{
СИНГУЛЯРНЫЕ ОРБИТЫ КОПРИСОЕДИНЕННОГО ПРЕДСТАВЛЕНИЯ ЕВКЛИДОВЫХ ГРУПП
}

\author{
А. М. Боярский, Т. В. Скрыпник
}

В работе предлагается метод вложения некоторых типов сингулярных орбит коприсоединенного представления групп $E(n)$ в пространство, дуальное к соответствующей алгебре Ли, в виде совместных поверхностей уровня полиномов специального вида.

Как известно, $E(n)=S O(n) \times \mathbb{R}^{n}$ может быть реализована как матричная группа следующим образом:

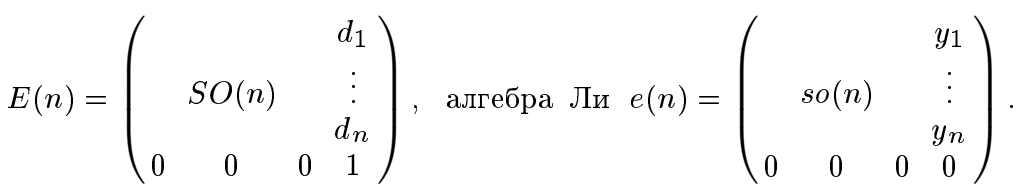

Будем задавать элемент $f \in e^{*}(n)$ в виде пары $f=(\mu, \nu)$, где $\mu \in s o^{*}(n)=s o(n), \nu \in \mathbb{R}_{n}^{*}=\mathbb{R}_{n}$. Тогда $a d_{(x, y)}^{*}(\mu, \nu)=\left([x, \mu]-\frac{1}{2} y \wedge \nu, x^{T}(\nu)\right)$. Известно [1], что все орбиты коприсоединенного действия группы $E(n)$ делятся на полупростые - устроенные как орбиты $S O(n-1)$ (см. [2]), и проходящие через точки с ненулевым $y$ - топологически устроенные как расслоения с базой - кокасательным расслоением над орбитой $S O(n)$ в $\mathbb{R}^{n}$ и слоем - орбитами ее стабилизатора. То есть $O_{(\mu, \nu)}=\left(E, T^{*}(S O(n) / S O(n-1)), O_{S O^{*}(n-1)}\right)=\left(E, T^{*} S_{\nu}^{n-1}, O_{S P^{*}(n-1)}\right)(\mu)$. Для описания явного вложения различных типов орбит в алгебры необходимо изучить структуру функций Казимира. В работе [2] было показано, что полньй набор примитивных инвариантов ортогональных групп можно выбрать так, чтобы каждый инвариант являлся суммой полных квадратов полиномов специального вида, а именно:

$$
p_{n-k}=\sum_{1 \leqslant i_{1}<\cdots<i_{k} \leqslant n} P f_{i_{1} \ldots i_{k}}^{2}(X), \quad \text { где } X \in \operatorname{so}(n)=\left\{X \in \operatorname{Mat}(n, R): X^{T}+X=0\right\},
$$

$P f_{i_{1} \ldots i_{k}}(X)$ - пфаффиан матрицы $X$ без $i_{1}, \ldots, i_{k}$ строчек и столбцов. Поскольку алгебра $e(n)$ может быть получена из $s o(n+1)$ контракцией, можно использовать результаты работы [3] o структуре функций Казимира контрактированных алгебр:

Лемма 1. Полнъцй набор функций Казимира группь $E(n)$ имеет вид:

$$
\begin{gathered}
p_{n-k}(x, y)=\sum_{1 \leqslant i_{1}<\cdots<i_{k} \leqslant n}\left(\sum_{1 \leqslant j \leqslant n} P f_{i_{1} \ldots j \ldots i_{k}}(X) y_{j}\right)^{2}, \\
\text { əде } X \in s o^{*}(n) \simeq s o(n), \quad y \in \mathbb{R}^{n *}=\mathbb{R}^{n} .
\end{gathered}
$$

ДокАЗАТЕЛЬСтво. Достаточно применить метод "контрактирования" инвариантов, описанный в [3], к коэффишиентам характеристического полинома матрицы $\left(\begin{array}{cc}X & \vec{y} \\ -\vec{y} & 0\end{array}\right) \in s^{*}(n+1)$ и учесть, что для пфаффианов существует, как и для детерминантов, формула разложения по строке и столбцу.

Интересно отметить, что все функции Казимира будут квадратичными по "абелевым" координатам. Легко видеть, что при $p_{n-k}=0$ автоматически имеем $\sum_{1 \leqslant j \leqslant n} P f_{i_{1} \ldots j \ldots i_{k}} y_{j} \equiv$ $P f_{i_{1} \ldots i_{k}}(X, y)=0, i_{k}=\overline{1, n}$, и размерность орбиты падает, так как эта орбита имеет новые инварианты, являющиеся ковариантами коприсоединенного представления.

Лемма 2. Уравнение $P f_{i_{1} \ldots i_{k}}(X, y)=0$ задает $A d_{E(n)}^{*}$ - инвариантное подмногообразие в $e^{*}(n)$. 
ДокАЗАТЕльСтво. Этот факт легко получить, применив процедуру контракции к формулам преобразования $P f_{i_{1} \ldots i_{k}}(X, y)$ под действием $a d_{S O(n+1)}^{*}$.

Для того чтобы отождествить такие инвариантные многообразия с орбитами коприсоединенного представления, заметим, что любой ковектор $(X, y)$ может быть переведен преобразованиями группы в ковектор вида $(X, 1,0, \ldots, 0)$, поэтому достаточно исследовать только точки такого вида и рассмотреть тип орбит, проходящих через них. На указанной плоскости уравнения принимают вид $P f_{i_{1}} \ldots 1 \ldots i_{k}=0$, и задача сводится к решенной в [2] задаче для $S O(n-1)$.

Теорема 1. Рассмотрим алгебру е $(d)$, реализованную матрицами вида

$$
\left(\begin{array}{cc}
X & y \\
0 & 0
\end{array}\right), \quad \text { əдe } \quad X \in s o(d), \quad y \in \mathbb{R}^{d}
$$

Тогда уравнения

$$
\left\{\begin{array}{l}
p_{2}=c_{2}, \\
\ldots \ldots \ldots \ldots \ldots \ldots \ldots \ldots \ldots \ldots \ldots \ldots \\
p_{2 n-2 k-2}=c_{2 n-2 k-2}, \\
\sum_{1 \leqslant j \leqslant n} P f_{i_{1} \ldots j \ldots i_{l}}(X) y_{j}=0,
\end{array}\right.
$$

əде $l=2 k$ при $d=2 n-1, l=2 k+1 n p u d=2 n, p_{d-a}-$ функции Казимира для группь $E(d)$, получаемье при помощи контракции, описанной в [3], из полного набора функций Казимира группь $S O(d+1) p_{d-a}^{(n+1)}-$ коэффициентов характеристического многочлена: $\operatorname{det}\left(\left(\begin{array}{cc}X & y \\ -y & 0\end{array}\right)-\lambda I\right)=\sum_{a} p_{d-a}^{(n+1)} \lambda^{a}, a P f_{i_{1} \ldots j \ldots i_{l}}(X)=\left(\operatorname{det} X_{i_{1} \ldots j \ldots i_{l}}\right)^{1 / 2}-n \oint a \phi \phi u-$ ан матрицы $X_{i_{1} \ldots j \ldots i_{l}}$, полученной из $X$ вычеркиванием $i_{1}, \ldots, i_{l}, j$ строк и столбцов, задают вложсение в е $(d)$ следующих типов сингулярных орбит коприсоединенного представления группь $E(d)$ :

1) $E(d) / \mathbb{R} \times S O(2(k+1)) \times S O(2)^{n-k-2}, d=2 n-1$,

2) $E(d) / \mathbb{R} \times S O(2(k+1)+1) \times S O(2)^{n-k-2}, \quad d=2 n$.

ДокАЗАТЕЛЬСтво. Стабилизатор точки плоскости $(X, 1,0, \ldots, 0)$, удовлетворяющей уравнению $P f_{i_{1} \ldots 1 \ldots i_{k}} y_{j}=0$, является пересечением стабилизатора $(1,0, \ldots, 0)$, т.е. $\mathbb{R} \times S O(d-1)$, со стабилизатором матрицы $X_{i_{1} \ldots 1} \ldots i_{l}$ из $S O(d-1)$, и задача сводится к решенной в [2].

Отметим, что орбиты минимальной размерности - кокасательные расслоения над сферой описываются только квадратичными функциями: квадратичной функцией Казимира $p_{2}$ и квадратичными пфофффианами. Последние, например, для группы $E(4)$ имеют вид $P_{i}=\varepsilon_{i j k l} x_{j k} y_{l}$ и являются евклидовыми аналогами векторов Любанского-Паули. При $n>4$ их структура аналогична. Система уравнений, описанная в теореме, имеет для $E(4)$ вид, аналогичный случаю $S O(5):[\vec{a} \times \vec{b}]+\vec{c} d=0,(\vec{a}, \vec{c})=0$, где $\vec{a}, \vec{b}, \vec{c}, d$ - три трехмерных вектора и скаляр, параметризующие $S O(5)$ и соответственно $E(4)$ (см. [2]).

\section{СПИСОК ЛИТЕРАТУРЫ}

[1] Трофимов В. В., Фоменко А. Т. // УМН. 1984. Т. 39. № 2(236). С. 3-56. [2] Боярский А., Скрыпник Т. // УМН. 1996. Т. 51. №3(309). С. 181-182; Боярский А., Скрыпник Т. // Укр. матем. журн. 1997. Т. 49. № 7. С. 895-906. [3] Рейман А. Г., СеменовТянШанский М. А. // Фундаментальные направления. Т. 16. М.: ВИНИТИ, 1987. С. 145-147.

Spiniza Institute, Utreht, the Netherlands; Bogolyubov Institute for Theoretical Physics 